\title{
Association of Pediatric Heart Transplant Coronary Vasculopathy with Abnormal Hemodynamic Measures
}

\author{
Ranjit Aiyagari, MD, Melisa Nika, MD, James G. Gurney, PhD, Janet E. Donohue, MPH, \\ Mary C. Zamberlan, CPNP, Karen King, MS, Dennis C. Crowley, MD, and Robert J. Gajarski, MD \\ C.S. Mott Children's Hospital, Division of Pediatric Cardiology, University of Michigan Health Center, Ann Arbor, MI, USA
}

\begin{abstract}
A B S T R A C T
Objective. Transplant coronary artery disease (TCAD) is the limiting factor to long-term cardiac allograft survival; however, presymptomatic diagnosis remains challenging. To that concern, we evaluated the association of abnormal catheter-derived filling pressures with TCAD in pediatric heart transplant (HTx) recipients.

Design, Patients, Outcome Measures. Data from 52 presymptomatic pediatric HTx patients were analyzed. Catheter-derived right ventricular end-diastolic pressure (RVEDP) and pulmonary capillary wedge pressure (PCWP) were recorded. Biopsies were collected to verify the absence of rejection.

Results. TCAD was diagnosed an average of 8.3 years post-HTx in 20 (38\%) patients, six of whom died and four of whom underwent retransplantation. Catheter-derived pressure measurements showed that RVEDP was elevated in TCAD compared with non-TCAD patients $(9.5 \pm 6.0$ vs. $5.4 \pm 4.7 ; P=.005)$, as was the PCWP $(12.9 \pm 5.7$ vs. $9.1 \pm 5.7 ; P=.012)$. Results from logistic regression analysis showed RVEDP $>10 \mathrm{~mm} \mathrm{Hg}$ or PCWP $>12 \mathrm{~mm} \mathrm{Hg}$ was associated with TCAD $(\mathrm{OR}=5.2 ; P=.010)$.

Conclusions. In this series, elevated ventricular filling pressures measured during routine surveillance catheterizations were associated with angiographic TCAD. Recognizing the association between elevated RVEDP/PCWP and TCAD may prompt earlier diagnosis and treatment of this potentially lethal process.
\end{abstract}

Key Words. Pediatric Transplant Coronary Vasculopathy

\section{Introduction}

$\mathrm{H}$ eart transplantation (HTx) is standard therapy for end-stage pediatric heart failure with a conditional survival of $50 \%$ at 13 years. ${ }^{1,2}$ The primary factor currently limiting longer-term graft survival is transplant coronary artery disease (TCAD) characterized by progressive coronary luminal obliteration, which in part represents an immune host response to newly recognized or upregulated antigens expressed on donor endothelial cells.

With the exception of a few recently developed immunosuppressive agents which may slow but likely not reverse this indolent process, retransplantation is the only option for these patients. Because TCAD mortality is high, ${ }^{3}$ timely diagnosis is critical. An estimated $31 \%$ of all deaths occurring more than 1 year posttransplantation are related to TCAD. ${ }^{4}$

Conventional diagnosis is based on selective coronary angiography, which is neither adequately sensitive nor specific. This modality generally fails to detect luminal narrowing of diffuse, smaller myocardial branches, and identifies only significantly obstructive lesions as progression of the disease effects anatomically larger epicardial vessels. ${ }^{5,6}$ Intravascular ultrasound (IVUS) is the only diagnostic strategy which has been shown to accurately predict extent and progression of disease, but IVUS is invasive and has inherent risks, particularly in children, and thus not routinely used in posttransplant surveillance procedures. ${ }^{5}$ Several other modalities remain largely investigational, including dobutamine stress echocardiography, ${ }^{6,7}$ coronary flow reserve measurement by Doppler 
flow wire ${ }^{8}$ or contrast echocardiography, ${ }^{9}$ and nuclear perfusion scans. ${ }^{10}$

Our recent anecdotal experience suggests that patients with significant and progressive TCAD appear to have elevated ventricular filling pressures measured during routine catheterizations for posttransplant biopsy and surveillance coronary angiography, suggestive of diastolic dysfunction. To date, there have been few investigations evaluating these hemodynamic measures with the angiographic presence of TCAD in presymptomatic patients. ${ }^{11}$ The purpose of this study was to evaluate the association of abnormal catheterderived filling pressures with TCAD in pediatric HTx recipients.

\section{Methods}

This retrospective study was approved by the Institutional Review Board (IRB) and was Health Insurance Portability and Accountability Act (HIPAA) compliant.

\section{Patient Demographics}

Data from catheterizations performed between 1990 and 2008 were reviewed for all patients transplanted after 1990. Demographic data including current age, age at HTx, and follow-up time was recorded.

\section{Institutional Norms}

For the early part of the study period, (1990-2000) coronary angiography was generally performed for the clinical indication of unexplained ventricular dysfunction without rejection on endomyocardial biopsy. For the later part of the study period, (2001-2008) HTx patients without known TCAD routinely underwent coronary angiography every 2 years. All patients reported in this series underwent coronary angiography at least once.

\section{Catheterization Data}

Selective angiograms from all patients who underwent surveillance coronary angiography were reviewed, and patients were categorized into two groups based on the presence or absence of angiographic TCAD. Using standard techniques, four to five endomyocardial biopsy specimens were collected, placed in formalin, and sent to a cardiac pathologist for grading to exclude concurrent treatable rejection.

Recorded data from the catheterization measurements included: right and left ventricular end-diastolic pressures (using pulmonary capillary wedge pressure [PCWP] as a surrogate for left ventricular end-diastolic pressure [LVEDP] in the absence of mitral valve disease), mean pulmonary artery pressure, and presence or absence of TCAD. Selective coronary angiograms were reviewed independently by two physicians (RJG and DCC) experienced in the interpretation of TCAD angiograms. Abnormal vessels identified by angiography were then identified according to the Stanford grading scale. ${ }^{12}$

\section{Statistical Analysis}

Demographic data were expressed as mean with standard deviation or median with range. Patients were analyzed in two groups: (1) those with vs. without TCAD, and (2) those who were retransplanted or died vs. those who were alive without retransplantation. Catheterization-derived pressures were compared between groups using the two-tailed $t$-test, applying the Satterthwaite method in the case of unequal variances. Logistic regression analysis was conducted to evaluate measures potentially associated with TCAD and death/retransplantation in univariate analysis. Statistical significance was defined as a $P$-value $\leq .05$.

\section{Results}

We identified 62 HTx who had coronary angiography performed between 1990 and 2008. Hemodynamic data were unavailable for six HTx patients. Of the remaining 56 recipients, 53 were considered eligible for the study. Hemodynamic data obtained in the presence of cellular rejection above grade $2 \mathrm{R}$ were excluded in three patients, including one patient whose only catheterization showed both TCAD and rejection. The patients' median age at initial transplant was 6.8 years. The overall survival for the cohort was $77 \%$ at a median of 6.8 years after initial heart transplant. A total of seven patients (13\%) underwent retransplantation, five because of documented TCAD and two because of rejection without documented TCAD.

Transplant coronary artery disease was diagnosed in 20 of 52 patients (38\%). At the time of TCAD diagnosis, 13 (65\%) had one affected vessel, four (20\%) had two affected vessels, and three $(15 \%)$ had three affected vessels. All patients with TCAD had left coronary disease and 30\% had right coronary disease as well. Eighteen patients $(90 \%)$ had Stanford B lesions, while eight (40\%) had type A lesions, and only two (10\%) had 
type C lesions. Nearly $20 \%$ of catheterizations within 2 years of transplant showed TCAD; beyond this point, there was a steady increase with time (Figure 1).

To compare the two groups of patients, we used data from the time of TCAD diagnosis, or the most recent data for those without TCAD (Table 1). Patients with TCAD were older at transplant (10.1 vs. 6.9 years, $P=.05)$ and further out from transplant (8.3 vs. 4.9 years, $P=.0003$ ). Mean right ventricular end-diastolic pressure (RVEDP) was $9.4 \mathrm{~mm} \mathrm{Hg}$ in patients with TCAD vs. 5.4 in patients without TCAD $(P=.008$, Figure 2). Mean PCWP was $12.9 \mathrm{~mm} \mathrm{Hg}$ in patients with TCAD vs. $9.1 \mathrm{~mm} \mathrm{Hg}$ in patients without TCAD $(P=.023$, Figure 2$)$. The association between high RVEDP $(>10 \mathrm{~mm} \mathrm{Hg}$ ) or PCWP $(>12 \mathrm{~mm} \mathrm{Hg})$ and concurrent TCAD was

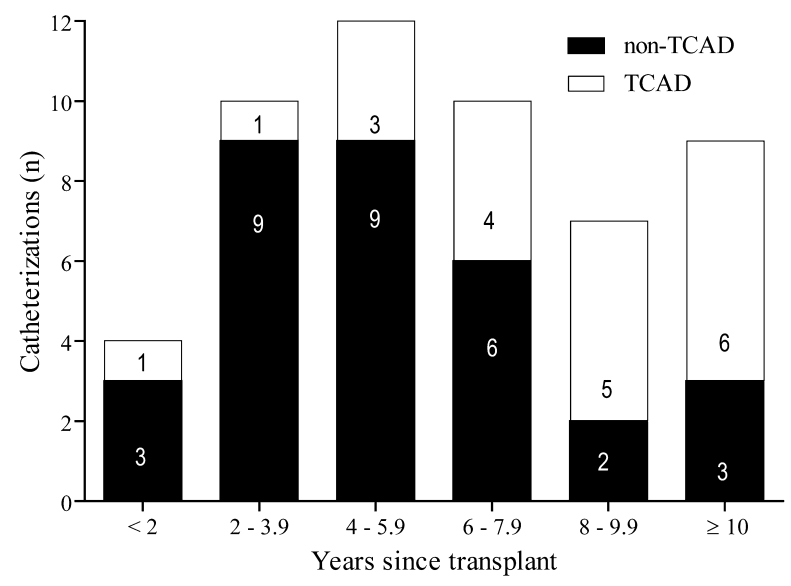

Figure 1. Number of routine catheterizations performed on post-heart transplant patients which showed coronary vasculopathy vs. time. Patients were censored at the time of diagnosis of TCAD. TCAD, transplant coronary artery disease. modeled using logistic regression. The odds of TCAD were 5.2 times higher in patients with elevations in one or both filling pressures $(95 \%$ confidence interval $[\mathrm{CI}]: 1.4-19.0 ; P=.010)$, compared with patients with no elevations. Adjusting for age at transplant did not result in a substantive change in this odds ratio. Adjusting for time since transplant, this odds ratio increased to $6.0(95 \%$ CI: $1.3-26.8 ; P=.020)$. The odds of TCAD were 1.4 times higher with each year posttransplant (95\% CI: $1.1-1.8 ; P=.003)$. Mean pulmonary artery pressures did not differ substantively between the groups $(18.9 \mathrm{~mm} \mathrm{Hg}$ vs. $16.5 \mathrm{~mm} \mathrm{Hg}, P=.180$ ).

Patients were also divided into those who subsequently died or were retransplanted (D/R), vs. those who were still alive and not retransplanted (A/NR; Table 2). For this analysis, the most recent

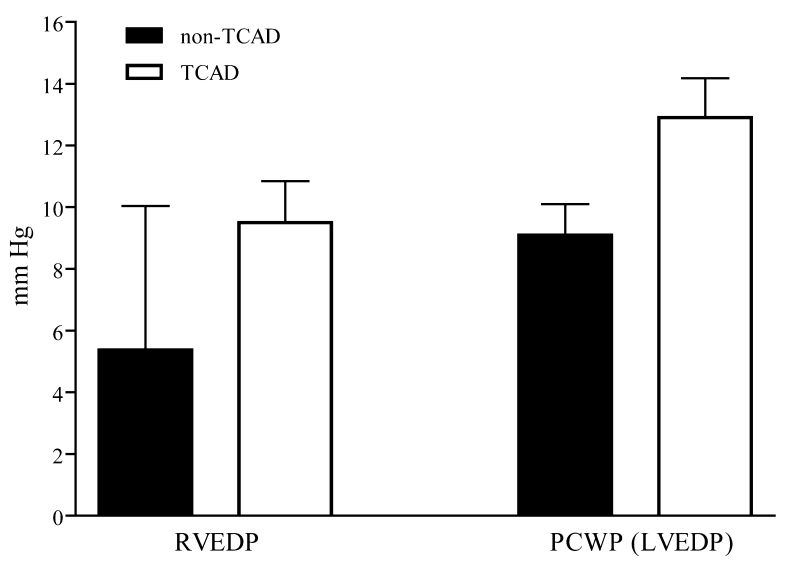

Figure 2. Mean RVEDP and PCWP for patients without and with coronary vasculopathy. Differences in both RVEDP and PCWP were statistically significant $(P=.008$ and $P=.023$, respectively). Error bars indicate standard deviation. TCAD, transplant coronary artery disease; RVEDP, right ventricular end-diastolic pressure; PCWP, pulmonary capillary wedge pressure; LVEDP, left ventricular end-diastolic pressure; $\mathrm{mm} \mathrm{Hg}$, millimeters of mercury.

Table 1. Hemodynamic data for patients without and with coronary vasculopathy

\begin{tabular}{|c|c|c|c|c|c|c|c|}
\hline & \multicolumn{2}{|c|}{ Overall } & \multicolumn{2}{|c|}{ Non-TCAD } & \multicolumn{2}{|c|}{ TCAD } & \multirow[b]{2}{*}{$P$} \\
\hline & $\mathrm{n}$ & Mean (SD) & $\mathrm{N}$ & Mean (SD) & $\mathrm{n}$ & Mean (SD) & \\
\hline Patient age at transplant (y) & 52 & $8.1(5.7)$ & 32 & $6.9(5.5)$ & 20 & $10.1(5.5)$ & .053 \\
\hline Time since transplant $(\mathrm{y})$ & 52 & $6.2(3.5)$ & 32 & $4.9(2.8)$ & 20 & $8.3(3.5)$ & $<.001$ \\
\hline $\operatorname{RVEDP}(\mathrm{mm} \mathrm{Hg})$ & 51 & $7.0(5.6)$ & 31 & $5.4(4.7)$ & 20 & $9.5(6.0)$ & .008 \\
\hline PCWP $(\mathrm{mm} \mathrm{Hg})$ & 52 & $10.6(5.9)$ & 32 & $9.1(5.7)$ & 20 & $12.9(5.7)$ & .023 \\
\hline Mean PAP (mm Hg) & 48 & $17.4(6.0)$ & 31 & $16.5(5.7)$ & 17 & $18.9(6.3)$ & .180 \\
\hline $\mathrm{EF}(\%)$ & 43 & $63.9(10.6)$ & 27 & $64.2(9.9)$ & 16 & $63.3(12.1)$ & .803 \\
\hline
\end{tabular}

Overall study population is shown in the left column. Differences were statistically significant for time since transplant, RVEDP, and PCWP. TCAD, transplant coronary artery disease; RVEDP, right ventricular end-diastolic pressure; PCWP, pulmonary capillary wedge pressure; PAP, pulmonary artery pressure; mm Hg, millimeters of mercury; SD, standard deviation; EF, left ventricular ejection fraction. 
Table 2. Hemodynamic data for patients who were alive and not retransplanted vs. those who died or been retransplanted

\begin{tabular}{|c|c|c|c|c|c|}
\hline & \multicolumn{2}{|c|}{ Alive/no re-HTx } & \multicolumn{2}{|c|}{ Died/re-HTx } & \multirow[b]{2}{*}{$P$} \\
\hline & $\mathrm{n}$ & Mean (SD) & $\mathrm{n}$ & Mean (SD) & \\
\hline Patient age at transplant $(\mathrm{y})$ & 36 & $7.8(5.8)$ & 16 & $9.0(5.6)$ & 0.476 \\
\hline Time since transplant $(\mathrm{y})$ & 36 & $6.5(3.5)$ & 16 & $6.4(4.0)$ & 0.933 \\
\hline $\operatorname{RVEDP}(\mathrm{mm} \mathrm{Hg})$ & 35 & $5.1(2.7)$ & 16 & $10.4(7.7)$ & 0.015 \\
\hline PCWP (mm Hg) & 36 & $9.1(3.9)$ & 16 & $13.7(7.7)$ & 0.037 \\
\hline Mean PAP $(\mathrm{mm} \mathrm{Hg})$ & 34 & $16.9(4.9)$ & 14 & $19.0(8.8)$ & 0.416 \\
\hline $\mathrm{EF}(\%)$ & 32 & $65.6(7.0)$ & 11 & $60.3(15.1)$ & 0.285 \\
\hline
\end{tabular}

Differences were statistically significant only for RVEDP and PCWP. HTx, heart transplant; RVEDP, right ventricular end-diastolic pressure; PCWP, pulmonary capillary wedge pressure; PAP, pulmonary artery pressure; $\mathrm{mm} \mathrm{Hg}$, millimeters of mercury; SD, standard deviation; EF, left ventricular ejection fraction.

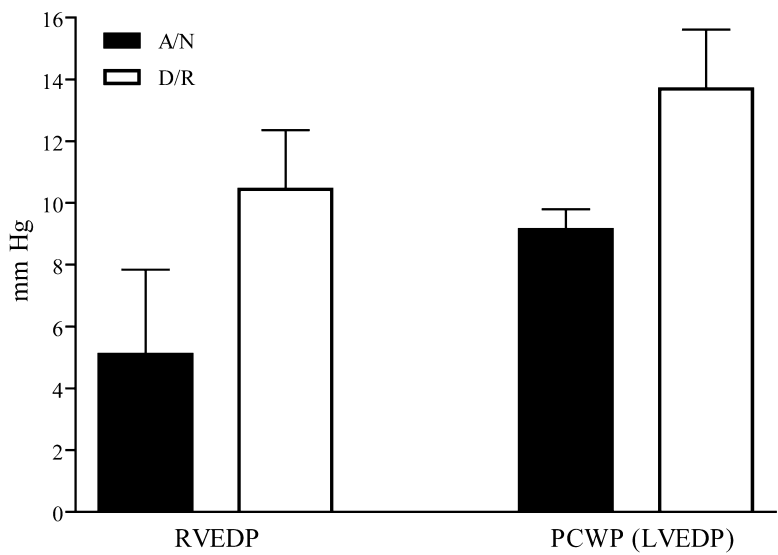

Figure 3. Mean RVEDP and PCWP for patients who were alive and not retransplanted $(\mathrm{A} / \mathrm{N})$ at last follow-up vs. patients who had died or been retransplanted (D/R). Differences in both RVEDP and PCWP were statistically significant $(P=.015$ and $P=.037$, respectively). Error bars indicate standard deviation. RVEDP, right ventricular end-diastolic pressure; PCWP, pulmonary capillary wedge pressure; LVEDP = left ventricular end-diastolic pressure; $\mathrm{mm} \mathrm{Hg}$, millimeters of mercury.

complete set of hemodynamic measurements, prior to retransplantation if applicable, was used. Mean RVEDP was $10.4 \mathrm{~mm} \mathrm{Hg}$ in D/R patients, vs. 5.1 in $\mathrm{A} / \mathrm{NR}$ patients $(P=.015$, Figure 3$)$. Mean PCWP was $13.7 \mathrm{~mm} \mathrm{Hg}$ in $\mathrm{D} / \mathrm{R}$ patients, vs. $9.1 \mathrm{~mm} \mathrm{Hg}$ in $\mathrm{A} / \mathrm{NR}$ patients $(P=.037$, Figure 3$)$. Results from logistic regression showed a 4.0 increased odds of subsequent death or retransplant for patients with elevated RVEDP and/or PCWP (95\% CI: $1.1-14.3 ; P=.032$ ), compared with patients without such elevations. There were no statistical differences between groups in age or time since transplant.

\section{Discussion}

These data suggest that right and left ventricular diastolic pressures are elevated at the time of
TCAD diagnosis. This is important because, despite the presence of established criteria, ${ }^{12}$ angiographic diagnosis of TCAD remains highly variable and operator-dependent. Conversely, RVEDP and PCWP can be measured quite accurately in nearly any patient with venous access, and their measurement adds little extra time to a right heart catheterization done for endomyocardial biopsy.

Our data are consistent with findings from Law and colleagues, who conducted a retrospective study of 18 TCAD patients with matched controls, finding mean RVEDP and PCWP of $11 \mathrm{~mm} \mathrm{Hg}$ and $14 \mathrm{~mm} \mathrm{Hg}$, respectively, in TCAD patients, vs. $6 \mathrm{~mm} \mathrm{Hg}$ and $8 \mathrm{~mm} \mathrm{Hg}$ in transplanted nonTCAD matched controls. ${ }^{11}$ Our study includes a larger group of patients followed over a longer period of time, and suggests that these hemodynamic generalizations seem to apply to retransplanted patients as well.

Our reported incidence of TCAD is higher than the general incidence reported in registry data. ${ }^{13}$ However, the patients included in this study are significantly older, and our data agree with those previously reported for older age groups. In addition, because of evolving practices and immunosuppressive regimens over the relatively long follow-up period, we would caution readers from using this series to determine the overall incidence of TCAD.

Recent evidence indicates that remodeling events leading to concentric hypertrophy are initiated as early as 1 week after heart transplantation. In addition, those patients with early concentric hypertrophy develop TCAD at a faster rate and have poorer graft survival. ${ }^{14}$ Right and left ventricular filling pressures are markers of diastolic function and increase with increasing degrees of hypertrophy. Given this, it is not surprising that an association between TCAD, RVEDP, and PCWP would be observed. Published studies involving prediction of PCWP in adult patients 
typically cite a PCWP of $15 \mathrm{~mm} \mathrm{Hg}$ or higher as a significant elevation; pressures below this are considered normal. ${ }^{15}$ In the current study, we found that filling pressures of greater than $10-12 \mathrm{~mm} \mathrm{Hg}$ were significant and were associated with TCAD in the pediatric heart transplant patients.

Early diagnosis remains a major challenge in combating TCAD and promoting graft longevity. In addition to their antirejection regimen, nearly all patients are already treated with a combination of agents designed to control hyperlipidemia, hyperglycemia, and hypertension. Most studies have demonstrated some correlation between hyperlipidemia and TCAD; ${ }^{16}$ however, it is less clear whether hyperglycemia and hypertension are independent risk factors for TCAD. ${ }^{17}$ The availability of agents such as sirolimus, which is known to slow the progression of TCAD in adult patients ${ }^{14,18}$ and results in prolonged survival in these patients, ${ }^{19}$ potentially provides a specific anti-TCAD pharmacologic therapy. Although prospective data in pediatric patients are lacking, it is possible that unfavorable changes in right and left ventricular enddiastolic pressures may guide the earlier use of this agent.

The chief limitation of this study is its retrospective nature, resulting in less than complete uniformity in data, especially early in the case series. As with all venous pressures, RVEDP and PCWP do vary with patients' volume status, and accurate hemodynamic tracings can be difficult to obtain in anxious or undersedated patients. Other limitations include the relatively small number of patients with complete hemodynamic data both before and after TCAD diagnosis, as well as operator dependence in coronary angiographic imaging and interpretation.

\section{Conclusions}

Right and left ventricular filling pressures are significantly elevated at the time of the diagnosis of transplant coronary artery disease. These elevations are associated with mortality and the need for retransplantation. Further study is necessary to determine if early pharmacologic intervention, based on these hemodynamic data, results in improved outcome in this population.

Corresponding Author: Ranjit Aiyagari, MD, University of Michigan, Division of Pediatric Cardiology, L1242 Women's, SPC 5204, 1500 E. Medical Center
Drive, Ann Arbor, MI 48109, USA. Tel: 734-647-8761; Fax: 734-936-9470; Email: ranjita@med.umich.edu

Grant support and conflicts to disclose: None.

Accepted in final form: September 29, 2010.

\section{References}

1 Boucek MM, Edwards LB, Keck BM, Trulock EP, Taylor DO, Hertz MI. Registry of the International Society for Heart and Lung Transplantation: eighth official pediatric report-2005. 7 Heart Lung Transplant. 2005;24:968-982.

2 Leman NR, Levi DS, Alejos JC, Wetzel GT. Predictors of graft longevity in pediatric heart transplantation. Pediatr Cardiol. 2005;26:762-767.

3 Pahl E, Zales VR, Fricker FJ, Addonizio LJ. Posttransplant coronary artery disease in children. A multicenter national survey. Circulation. 1994;90: II56-II60.

4 Mulla NF, Johnston JK, Vander Dussen L, et al. Late rejection is a predictor of transplant coronary artery disease in children. 7 Am Coll Cardiol. 2001; $37: 243-250$.

5 Costello JM, Wax DF, Binns HJ, Backer CL, Mavroudis C, Pahl E. A comparison of intravascular ultrasound with coronary angiography for evaluation of transplant coronary disease in pediatric heart transplant recipients. 7 Heart Lung Transplant. 2003; 22:44-49.

6 Di Filippo S, Semiond B, Roriz R, Sassolas F, Raboisson MJ, Bozio A. Non-invasive detection of coronary artery disease by dobutamine-stress echocardiography in children after heart transplantation. 7 Heart Lung Transplant. 2003;22:876-882.

7 Dipchand AI, Bharat W, Manlhiot C, Safi M, Lobach NE, McCrindle BW. A prospective study of dobutamine stress echocardiography for the assessment of cardiac allograft vasculopathy in pediatric heart transplant recipients. Pediatr Transplant. 2008; 12:570-576.

8 Schubert S, Abdul-Khaliq H, Wellnhofer E, et al. Coronary flow reserve measurement detects transplant coronary artery disease in pediatric heart transplant patients. 7 Heart Lung Transplant. 2008; 27:514-521.

9 Tona F, Caforio ALP, Montisci R, et al. Coronary flow velocity pattern and coronary flow reserve by contrast-enhanced transthoracic echocardiography predict long-term outcome in heart transplantation. Circulation. 2006;114:I49-I55.

10 Maiers J, Hurwitz R. Identification of coronary artery disease in the pediatric cardiac transplant patient. Pediatr Cardiol. 2008;29:19-23.

11 Law Y, Boyle G, Miller S, et al. Restrictive hemodynamics are present at the time of diagnosis of allograft coronary artery disease in children. Pediatr Transplant. 2006;10:948-952. 
12 Gao SZ, Alderman EL, Schroeder JS, Silverman JF, Hunt SA. Accelerated coronary vascular disease in the heart transplant patient: coronary arteriographic findings. 7 Am Coll Cardiol. 1988;12:334-340.

13 Kirk R, Edwards L, Aurora P, et al. Registry of the International Society for Heart and Lung Transplantation: eleventh official pediatric heart transplantation report-2008. I Heart Lung Transplant. 2008;27:970-977.

14 Raichlin E, Bae J-H, Khalpey Z, et al. Conversion to sirolimus as primary immunosuppression attenuates the progression of allograft vasculopathy after cardiac transplantation. Circulation. 2007;116:27262733.

15 Quinones M. Evaluation of intracardiac filling pressures. In: Klein AL, Garcia MJ, eds. Diastology:
Clinical Approach to Diastolic Heart Failure. Philadelphia, Pa: Saunders/Elsevier; 2008: 181-192.

16 Ventura HO, Mehra MR, Smart FW, Stapleton DD. Cardiac allograft vasculopathy: current concepts. Am Heart 7. 1995;129:791-799.

17 Hathout E, Beeson WL, Kuhn M, et al. Cardiac allograft vasculopathy in pediatric heart transplant recipients. Transpl Int. 2006;19:184-189.

18 Mancini D, Pinney S, Burkhoff D, et al. Use of rapamycin slows progression of cardiac transplantation vasculopathy. Circulation. 2003;108:48-53.

19 Zakliczynski M, Swierad M, Nozynski J, Maruszewski M, Zembala M. Survival benefit in heart transplant recipients who have coronary artery disease confirmed using angiography and are receiving sirolimus. Transplant Proc. 2009;41:285-288. 